\title{
Semiclassical Description of Anisotropic Magnets for Spin $S=1$
}

\author{
Khikmat Muminov and Yousef Yousefi \\ Physical-Technical Institute Named after S. U. Umaro, Academy of Sciences of Republic of Tajikistan, Aini Avenue 299/1, \\ Dushanbe, Tajikistan
}

Correspondence should be addressed to Yousef Yousefi, yousof54@yahoo.com

Received 10 August 2011; Revised 13 September 2011; Accepted 15 September 2011

Academic Editor: Roberto Zivieri

Copyright (c) 2012 K. Muminov and Y. Yousefi. This is an open access article distributed under the Creative Commons Attribution License, which permits unrestricted use, distribution, and reproduction in any medium, provided the original work is properly cited.

\begin{abstract}
Nonlinear equations describing one-dimensional non-Heisenberg ferromagnetic model are studied by the use of generalized coherent states in a real parameterization. Also, dissipative spin wave equation for dipole and quadruple branches is obtained if there is a small linear excitation from the ground state.
\end{abstract}

\section{Introduction}

In the past decades, magnets with spin value $s=1 / 2$ have been studied completely. There are dipoles, quadruples and higher-order branches that affect the behavior of magnet crystal. However, the only necessary tool for describing the behavior of this kind of magnets is dipole branch effect, and the order branches are not necessary. This results in linear approximation for describing the magnet behavior.

Indeed, only dipole branch effect has been used for describing magnets with spin value $s \geq 1$, and the effect of quadrupole and higher-order branches has been ignored. Recently, however, due to the new developments in mathematics and technology and also due to the great potential of quadrupole branch in description of nanoparticles, its important role cannot be ignored $[1,2]$.

Using the effects of both dipole and quadrupole branches results in a nonlinear approximation. The use of higherorder multipole effects yields more accurate approximations which demand more complicated equations. In this paper, only the effect of quadruple branch for Hamiltonians described by (1) is considered. Study of isotropic and anisotropic spin Hamiltonian with non-Heisenberg terms are complicated due to quadruple excitation dynamics [3-5]. Antiferromagnetic property of this excitation in states near the ground proves the existence of it. The effect of this calculation has been studied by Dzyaloshinskii [6]. The results obtained through the quadrupole excitation in nanoparticles
$\mathrm{Fe}_{8}$ and $\mathrm{Mn}_{12}$ are more in line with numerical calculations and laboratory results $[7,8]$.

In classical physics term, the number of parameters required for a full macroscopic description of the magnet behavior is equal to $4 s$, where $s$ is the spin value. Also realparameterized coherent states based on related group is used to obtain classical equation of motion and to describe multipole dynamics $[9,10]$. Here, Heisenberg ferromagnets with anisotropic term as described by (1) are considered:

$$
\widehat{H}=-J \sum_{i}\left(\overrightarrow{\widehat{S}}_{i}{\widehat{S_{i+1}}}+\delta \widehat{S}_{i}^{z} \widehat{S}_{i}^{z}\right) .
$$

Here, $\widehat{S}_{i}^{x}, \widehat{S}_{i}^{y}$, and $\widehat{S}_{i}^{z}$ are the spin operators acting at a site $i$, and $\delta$ is the anisotropy coefficient. This Hamiltonian is related to a one-dimensional ferromagnetic spin chains, and the coefficient $J$ is positive.

In order to calculate the effect of quadrupole excitation, first, the classical equivalent of Hamiltonian (1) is obtained and then, by analyzing such equation for small linear excitation from the ground states, the spin wave solution is found. This process requires the following steps.

(1) Obtaining coherent states for spin $s=1$ which are coherent states of $\mathrm{SU}(3)$ group.

(2) Calculating the average values of spin operator.

(3) Obtaining classical spin Hamiltonian equation using previously calculated values. 
(4) Computing Lagrangian equation by the use of Feynman path integral over coherent states and then computing classical equations of motion.

(5) For finding nonlinear equations of magnet behavior, it is necessary to substitute resulted Hamiltonian in classical equations of motion. Solutions of these nonlinear equations result in soliton description of magnet that is not needed here.

(6) Calculating ground states of magnet and then linearizing the nonlinear equations around the ground states for small excitation.

(7) At the end, calculating spin wave equation and dispersion equation.

In what follows, the mathematical descriptions of the above steps are presented.

\section{Theory and Calculation}

In quantum mechanics, coherent states are special kind of quantum states that their dynamics are very similar to their corresponding classical system. These states are obtained by act of Weil-Heisenberg group operator on vacuum state. Vacuum state of SU(3) group is $(1,0,0)^{T}$, and coherent state is introduced as [11]

$$
\begin{aligned}
|\psi\rangle & =D^{1 / 2}(\theta, \phi) e^{-i \gamma \widehat{S}^{z}} e^{2 i g \widehat{Q}^{x y}}|0\rangle \\
& =C_{0}|0\rangle+C_{1}|1\rangle+C_{2}|2\rangle,
\end{aligned}
$$

where $D^{1 / 2}(\theta, \phi)$ is Wigner function and $Q^{x y}$ is quadruple moment which is written in the following form:

$$
\widehat{Q}^{x y}=\frac{i}{2}\left(\begin{array}{ccc}
0 & 0 & 1 \\
0 & 0 & 0 \\
-1 & 0 & 0
\end{array}\right) .
$$

Coefficients $C_{0}$ to $C_{2}$ are computed from these equations

$$
\begin{gathered}
C_{0}=e^{i \phi}\left(e^{-i \gamma} \sin ^{2}\left(\frac{\theta}{2}\right) \cos g+e^{i \gamma} \cos ^{2}\left(\frac{\theta}{2}\right) \sin g\right), \\
C_{1}=\frac{\sin \theta}{\sqrt{2}}\left(e^{-i \gamma} \cos g-e^{i \gamma} \sin g\right), \\
C_{2}=e^{-i \phi}\left(e^{-i \gamma} \cos ^{2}\left(\frac{\theta}{2}\right) \cos g+e^{i \gamma} \sin ^{2}\left(\frac{\theta}{2}\right) \sin g\right) .
\end{gathered}
$$

Two angles, $\theta$ and $\phi$, determine the direction of classical spin vector in spherical coordinate system. The angle $\gamma$ determines the direction of quadruple moment around the spin vector, and parameter $g$ shows change of the magnitude of spin vector.

In order to obtain the classical equivalent of Hamiltonian (1), the classical equivalent of spin vector and its corresponding products should be computed. So, consider

$$
\vec{S}=\langle\psi|\overrightarrow{\widehat{S}}| \psi\rangle
$$

as classical spin vector, and also consider

$$
Q^{i j}=\left\langle\psi\left|\widehat{S}^{i} \widehat{S}^{j}\right| \psi\right\rangle
$$

as components of quadruple moment. Spin operators can be commute in different lattices; so

$$
\left\langle\psi\left|\widehat{S}_{n}^{i} \widehat{S}_{n+1}^{j}\right| \psi\right\rangle=\left\langle\psi\left|\widehat{S}_{n}^{i}\right| \psi\right\rangle\left\langle\psi\left|\widehat{S}_{n+1}^{j}\right| \psi\right\rangle,
$$

where $|\psi\rangle=|\psi\rangle_{n}|\psi\rangle_{n+1}$.

The average spin values in $\mathrm{SU}(3)$ group are defined as [12]

$$
\begin{gathered}
S^{+}=e^{i \phi} \cos (2 g) \sin \theta, \\
S^{-}=e^{-i \phi} \cos (2 g) \sin \theta, \\
S^{z}=\cos (2 g) \cos \theta, \\
S^{2}=\cos ^{2}(2 g) .
\end{gathered}
$$

Classical Hamiltonian can be obtained from the average calculation of Hamiltonian (1) over coherent states. The classical continuous limit of Hamiltonian in $\mathrm{SU}(3)$ group is

$$
\begin{aligned}
& H_{\mathrm{cl}} \\
& \begin{aligned}
=-J \int \frac{d x}{a_{0}}( & \cos ^{2}(2 g) \\
& +\frac{\delta}{2}\left(\cos ^{2} \theta+\sin (2 g) \cos (2 \gamma) \sin ^{2} \theta\right) \\
& \left.-\frac{a_{0}^{2}}{2}\left(\left(\theta_{x}^{2}+\phi_{x}^{2} \sin ^{2} \theta\right) \cos ^{2}(2 g)+4 g_{x}^{2} \sin ^{2}(2 g)\right)\right) .
\end{aligned}
\end{aligned}
$$

The above classical Hamiltonian is substituted in equation of motion that was obtained from the Lagrangian, and the result is classical equations of motion:

$$
\begin{aligned}
\frac{1}{\omega_{0}} \phi_{t}= & \delta \cos \theta(\sec (2 g)-\cos (2 \gamma) \tan (2 g)) \\
& +a_{0}^{2} \cos (2 g)\left(\theta_{x x} \csc \theta+\phi_{x}^{2} \cos \theta\right) \\
\frac{1}{\omega_{0}} \theta_{t}= & \frac{\delta}{2} \sin (2 \theta) \sin (2 \gamma) \tan (2 g)-a_{0}^{2} \phi_{x x} \cos (2 g) \sin \theta \\
\frac{1}{\omega_{0}} g_{t}= & -\frac{\delta}{2} \sin (2 \gamma) \sin ^{2} \theta \\
\frac{1}{\omega_{0}} \gamma_{t}= & (4 \cos (2 g) \\
& -\delta(\cos (2 \gamma)(\cot (4 g)-\cos (2 \theta) \csc (4 g)) \\
& \left.\left.+\cos ^{2} \theta \sec (2 g)\right)\right) \\
& +\left(\begin{array}{c}
\cos (2 g)\left(8 g_{x}^{2}-2 \theta_{x}^{2}\right. \\
\left.+\frac{1}{2} \phi_{x}^{2}(-3+\cos (2 \theta))-\theta_{x x} \cot \theta\right) \\
\left.+4 g_{x x} \sin (2 g)\right) a_{0}^{2} .
\end{array}\right.
\end{aligned}
$$


These equations describe nonlinear dynamics of non-Heisenberg ferromagnetic chain completely. Solutions of these equations are magnetic solitons that are not studied in this paper.

In this paper, only the linearized form of (10) for small excitation from the ground states is considered. To this end, first, classical ground states must be calculated. therefore in the above Hamiltonian, only nonderivative part is taken into account:

$$
\begin{aligned}
H_{0}=-J \int \frac{d x}{a_{0}}( & \cos ^{2}(2 g) \\
& \left.+\frac{\delta}{2}\left(\cos ^{2} \theta+\sin (2 g) \cos (2 \gamma) \sin ^{2} \theta\right)\right) .
\end{aligned}
$$

It is necessary to calculate derivative of (11) with respect to all variables to find out minimum of $H_{0}$. As a result, if $\delta<0$, ground states are at these points

$$
\theta=\frac{\pi}{2}, \quad \gamma=\frac{\pi}{2}, \quad \sin 2 g_{0}=\frac{|\delta|}{4}, \quad|\delta|<4 .
$$

In this paper, only dispersion of spin wave in neighborhood of the ground states is studied. For this purpose, small linear excitations from the ground states, as shown in (13), are defined:

$$
\begin{gathered}
\theta \longrightarrow \frac{\pi}{2}-\theta, \\
2 \gamma \longrightarrow \pi+\gamma, \\
2 g \longrightarrow g_{0}+g .
\end{gathered}
$$

In this situation, the linearized classical equations of motion are

$$
\begin{gathered}
\frac{1}{\omega_{0}} \phi_{t}=\delta\left(\sec g_{0}+\tan g_{0}\right) \theta+a_{0}^{2} \cos g_{0} \theta_{x x}, \\
\frac{1}{\omega_{0}} \theta_{t}=-a_{0}^{2} \phi_{x x} \cos g_{0}, \\
\frac{1}{\omega_{0}} g_{t}=-\frac{\delta}{2} \gamma, \\
\frac{1}{\omega_{0}} \gamma_{t}=-2\left(2 \sin g_{0}+\frac{\delta}{\cos g_{0}}\right) g+4 a_{0}^{2} g_{x x} \sin g_{0} .
\end{gathered}
$$

Consider functions $\theta, \phi, \gamma$, and $g$ as plane waves to obtain dispersion equation:

$$
\begin{aligned}
& \phi=\phi_{0} e^{i(\omega t-k x)}+\overline{\phi_{0}} e^{-i(\omega t-k x)}, \\
& \theta=\theta_{0} e^{i(\omega t-k x)}+\overline{\theta_{0}} e^{-i(\omega t-k x),} \\
& g=g_{0} e^{i(\omega t-k x)}+\overline{g_{0}} e^{-i(\omega t-k x)}, \\
& \gamma=\gamma_{0} e^{i(\omega t-k x)}+\overline{\gamma_{0}} e^{-i(\omega t-k x)} .
\end{aligned}
$$

Substitute of these equations in (14), then

$$
\begin{gathered}
\omega_{1}^{2}=\omega_{0}^{2} k^{2} a_{0}^{2}\left(\delta\left(1+\sin g_{0}\right)+k^{2} a_{0}^{2} \cos ^{2} g_{0}\right), \\
\omega_{2}^{2}=\omega_{0}^{2}\left[2 \sin g_{0} k^{2} a_{0}^{2}+\delta\left(\frac{4 \delta}{\sin ^{2} g_{0}}-2 \sin g_{0}\right)\right] .
\end{gathered}
$$

These equations are dispersion equations of spin wave near the ground states in SU(3) group.

\section{Conclusions}

In this paper, describing equations of one-dimensional anisotropic non-Heisenberg Hamiltonians are obtained using real-parameter coherent states. It was indicated that both dipole and quadruple excitations have different dispersion if there is small linear excitation from the ground state.

In addition, it was indicated, that for anisotropic ferromagnets, the magnitude of average quadruple moment is not constant and its dynamics consists of two parts. One part is rotational dynamics around the classical spin vector $\left(\gamma_{t} \neq 0\right)$ and the other related to the change of the magnitude of quadruple moment $\left(g_{t} \neq 0\right)$.

\section{References}

[1] E. L. Nagaev, "Anomalous magnetic structures and phase transitions in non-Heisenberg magnetic materials," Soviet Physics Uspekhi, vol. 25, p. 31, 1982.

[2] E. L. Nagaev, Magnets with Nonsimple Exchange Interactions, Nauka, Moscow, Russia, 1988.

[3] Kh. O. Abdulloev and Kh. Kh. Muminov, "Semiclassical description of anisotropic magnets acted upon by constant external magnetic fields," Physics of the Solid State, vol. 36, no. 1, pp. 93-97, 1994.

[4] B. A. Ivanov, A. Yu. Galkin, R. S. Khymyn, and A. Yu. Merkulov, "Nonlinear dynamics and two-dimensional solitons for spin $S=1$ ferromagnets with biquadratic exchange," Physical Review B, vol. 77, no. 6, Article ID 064402, 2008.

[5] Yu. A. Fridman, O. A. Kosmachev, A. K. Kolezhuk, and B. A. Ivanov, "Spin nematic and antinematic states in a spin-3/2 isotropic non-Heisenberg magnet," Physical Review Letters, vol. 106, no. 9, Article ID 097202, 4 pages, 2011.

[6] I. Dzyaloshinskii, "External magnetic fields of antiferromagnets," Solid State Communications, vol. 82, no. 7, pp. 579-580, 1992.

[7] G. Bellessa, E. Del Barco, N. Vernier et al., "Quantum coherence in $\mathrm{Fe}_{8}$ molecular nanomagnets," Europhysics Letters, vol. 47, no. 6, pp. 722-728, 1999.

[8] M. S. Foss-Feig and J. R. Friedman, "Geometric-phase-effect tunnel-splitting oscillations in single-molecule magnets with fourth-order anisotropy induced by orthorhombic distortion," Europhysics Letters, vol. 86, no. 2, Article ID 27002, 2009.

[9] V. S. Ostrovskii, Soviet Physics JETP, vol. 64, no. 5, p. 999, 1986.

[10] N. A. Mikushina and A. S. Moskvin, "Dipole and quadrupole skyrmions in $S=1$ (pseudo)spin systems," Physics Letters A, vol. 302, no. 1, pp. 8-16, 2002.

[11] Kh. O. Abdulloev and Kh. Kh. Muminov, "Coherent states of $\mathrm{SU}(4)$ group in real parameterization and Hamiltonian equations of motion," Reports of Tajikistan Academy of science, vol. 36, no. 6, I993 (Russian).

[12] Kh. O. Abdulloev and Kh. Kh. Muminov, "Accounting of quadrupole dynamics of magnets with spin," Proceedings of Tajikistan Academy of Sciences, no. 1, pp. 28-30, 1994 (Russian). 

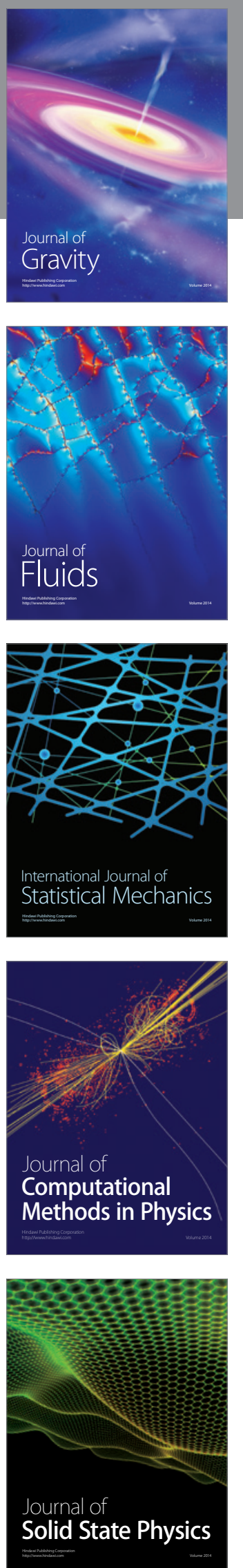

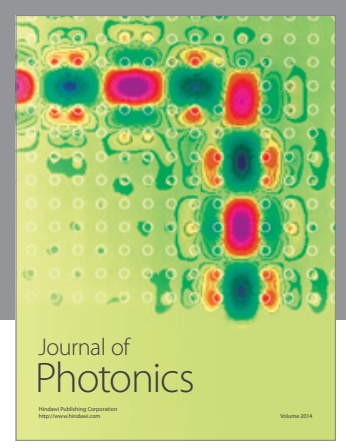

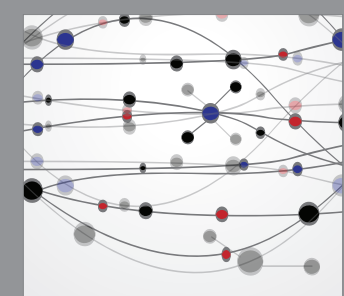

The Scientific World Journal
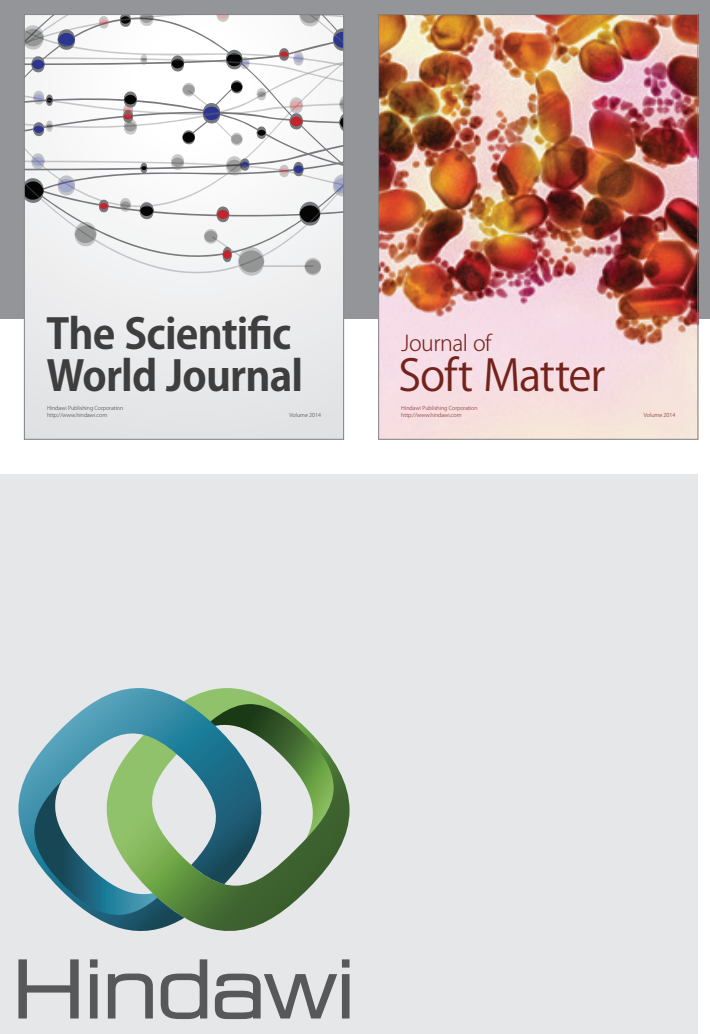

Submit your manuscripts at

http://www.hindawi.com
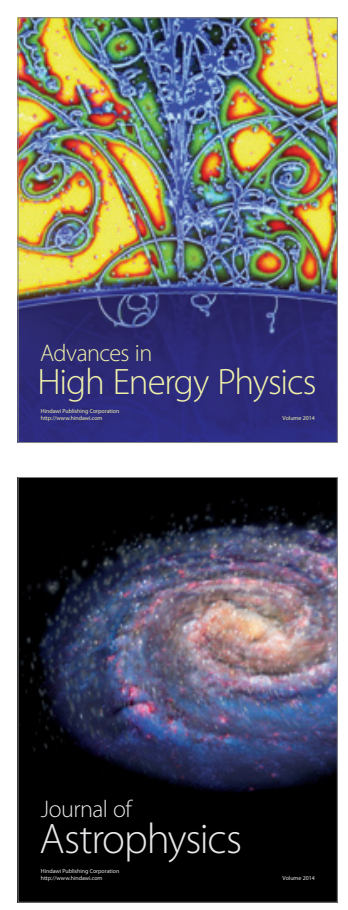
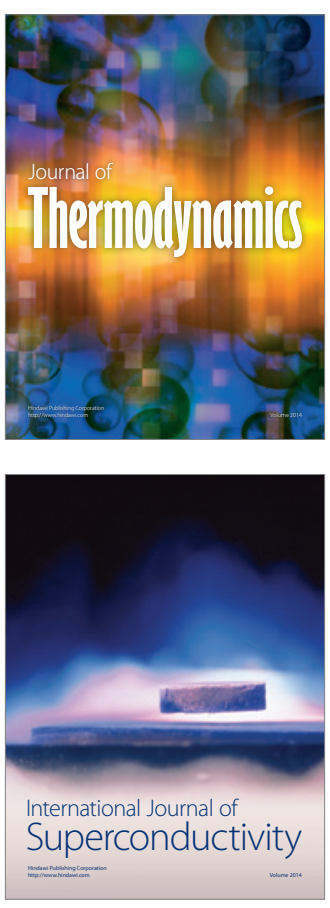
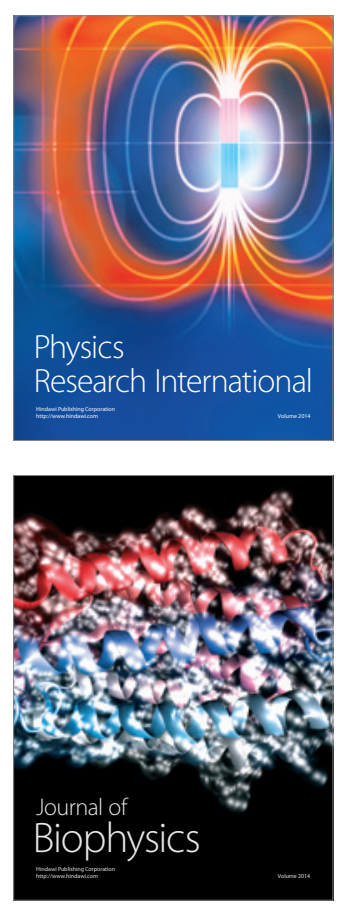
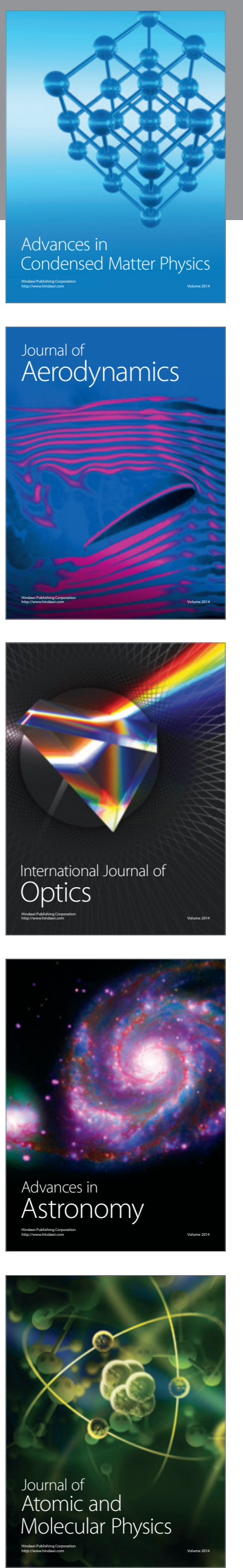